\title{
Erratum: Platelet-derived growth factor-a receptor is the cellular receptor for human cytomegalovirus gHgLgO trimer
}

Anna Kabanova, Jessica Marcandalli, Tongqing Zhou, Siro Bianchi, Ulrich Baxa, Yaroslav Tsybovsky, Daniele Lilleri, Chiara Silacci-Fregni, Mathilde Foglierini, Blanca Maria Fernandez-Rodriguez, Aliaksandr Druz, Baoshan Zhang,

Roger Geiger, Massimiliano Pagani, Federica Sallusto, Peter D. Kwong, Davide Corti, Antonio Lanzavecchia and Laurent Perez

Nature Microbiology 1, 16082 (2016); published 6 June 2016; corrected 20 June 2016.

The original version of this Letter contained several errors introduced during production. In Fig. 1d-f the asterisks indicating statistical significance have been rotated through $90^{\circ}$ so they are no longer obscured by the data bars. Fig. 3 has been modified so that the legends represent the correct concentrations (b, 0-100 nM; c, 0.3-25 nM). Additionally, the affiliations have been updated to distinguish that Antonio Lanzavecchia and Laurent Perez jointly supervised this work. We apologize to the authors and readers for these errors and any confusion caused. All versions of the Letter have been updated accordingly. 